\title{
Is neurocysticercosis a risk factor in coexistent intracranial disease? An MRI based study
}

\section{R Azad, R K Gupta, S Kumar, C M Pandey, K N Prasad, N Husain, M Husain}

Background: Previous reports have suggested that neurocysticercosis is associated with glioma and Japanese encephalitis, and that it is a risk factor for stroke.

Objective: To determine if neurocysticercosis has a significant association with, or is a risk factor for, coexistent pathologies such as Japanese encephalitis, glioma, abscess, tuberculoma, or infarction.

Subjects: 10350 patients from the hospital population who underwent $1.5 \mathrm{~T}$ cranial magnetic resonance imaging during the previous 12 years were evaluated for the presence of neurocysticercosis and coexisting pathology.

Design: Retrospective cohort analysis.

Results: The prevalence of neurocysticercosis in cases with dual pathology was significantly less than in a control group $11.1 \% \vee 8.3 \% ; z=11.05 ; p<0.001$, power of test $=1$ ). Neurocysticercosis lesions were less common $(p<0.05)$ in the different subgroups of coexistent pathology than in the control group except in the case of Japanese encephalitis, where the difference was nonsignificant $(z=0.69, p=0.49)$. The relative risk was less than 1 in all subgroups except Japanese encephalitis, where it was 1.23. The location of neurocysticercosis lesions and the presence of perilesional oedema did not affect coexistent lesion location or severity on a particular side ( $p=0.413$ and 0.623 for location and perilesional oedema, respectively). When the above factors were analysed separately in patients with Japanese encephalitis, they also did not affect coexistent lesion location or severity $(p=0.659$ and 0.548 , respectively)

Conclusions: The coexistence of neurocysticercosis and other lesions may be an incidental observation in a few patients referred from areas of high prevalence and endemicity. It appears unlikely that neurocysticercosis is a risk factor for other intracerebral pathology. The location of neurocysticercosis lesions and whether or not there is surrounding perilesional oedema do not appear to affect the location or severity of coexisting lesions.

$\mathrm{N}$ eurocysticercosis is the most common parasitic infection of the central nervous system. Owing to its frequent endemicity, various intracranial pathologies such as Japanese encephalitis, glioma, abscess, tuberculosis, and infarction may coexist with neurocysticercosis on imaging studies. ${ }^{1-7}$ There have been previous reports that neurocysticercosis has a significant association with glioma and Japanese encephalitis; in addition, neurocysticercosis has been claimed to be a risk factor for glioma, Japanese encephalitis, and stroke. ${ }^{1258}$ It has also been reported that neurocysticercosis leads to an asymmetrical distribution of coexisting lesions, such diseases being more likely to be on the side of the neurocysticercosis lesions and their severity greater in the hemisphere where the cysts are located, or where their number is greatest, or where there is associated perilesional oedema. ${ }^{25}$

We present a retrospective analysis of magnetic resonance imaging (MRI) in cases of neurocysticercosis with other common coexistent intracranial pathologies such as Japanese encephalitis, glioma, abscess, tuberculoma, and infarction. Our aim was to determine whether neurocysticercosis is significantly associated with, or is a risk factor for, such types of intracranial pathology. We also evaluated the relation between the presence of neurocysticercosis and surrounding perilesional oedema in a particular hemisphere and the location and severity of coexisting lesions.

\section{METHODS}

We carried out a retrospective assessment of 10350 MRI brain scans in patients referred to us at a tertiary care hospital during 1991 to 2002 and found 722 cases of neurocysticercosis. The age of the patients ranged from 6 to 73 years (mean 30.3 years). The sex ratio (male to female) was 1.09. The study group consisted of 21 patients in whom a coexisting lesion was found. Among these there were 10 cases of Japanese encephalitis (of a total of 98 cases of Japanese encephalitis), four of glioma (of a total of 796), three of abscess (of a total of 116), two of tuberculoma (of a total of 360), and two of infarction (of a total of 515). The age of the patients with coexistent disease ranged from 8 to 60 years (mean 28.2 years). The male to female ratio was 1.1.

A control group was formed consisted of the remaining 8465 patients (age group range 6 to 73 years, mean 29.6 years; sex ratio 1.09) who, on imaging, were either normal or had intracranial pathology different from the study group. There were 701 cases of neurocysticercosis in the control population.

The cases with coexisting lesions came mostly from areas in Eastern Uttar Pradesh and Bihar States of India, where the general level of sanitation is poor and rearing of pigs prevalent. These patients were by and large of low socioeconomic status. The clinical symptoms were non-specific, ranging between fever, headache, seizures, neck rigidity, altered sensorium, coma, and focal neurological deficit.

The diagnosis of neurocysticercosis was based on objective clinical, imaging, immunological, and epidemiological data, in accordance with the latest recommendations. ${ }^{9}$ The absolute criterion on neuroimaging is the presence of cystic lesions showing the scolex, while major criteria are cystic lesions highly suggestive of neurocysticercosis without a scolex, single or multiple ring or nodular enhancing lesions, and parenchymal round calcifications. The images were interpreted for the presence of these criteria by two experienced radiologists. Glioma, abscess, and tuberculoma were diagnosed on the basis of imaging, histopathology, and follow up on treatment. The diagnosis of Japanese encephalitis was based on epidemiological, clinical, serological $(n=98)$, and imaging criteria. Infarction was diagnosed on the basis of the clinical course and imaging features. 
Table 1 Summary of the results

\begin{tabular}{|c|c|c|c|c|c|c|c|c|}
\hline \multirow[b]{2}{*}{ Pathology } & \multirow[b]{2}{*}{$\begin{array}{l}\text { Number } \\
\text { of cases }\end{array}$} & \multicolumn{2}{|c|}{ Proportion with NCC } & \multicolumn{2}{|c|}{$\begin{array}{l}\text { Statistical } \\
\text { significance }\end{array}$} & \multirow[b]{2}{*}{$\begin{array}{l}\text { Power } \\
\text { of test }\end{array}$} & \multirow[b]{2}{*}{ RR } & \multirow[b]{2}{*}{$95 \% \mathrm{Cl}$ of $\mathrm{RR}$} \\
\hline & & Cases (n) & $\begin{array}{l}\text { Controls } \\
(n=8465)\end{array}$ & $\mathrm{z}$ Value & $\mathrm{p}$ Value & & & \\
\hline Overall & 1885 & $0.011(21)$ & $0.083(701 *)$ & 11.05 & $<0.001$ & 1.0 & 0.13 & 0.09 to 0.21 \\
\hline JE & 98 & $0.102(10)$ & 0.083 & 0.69 & 0.49 & 0.6 & 1.23 & 0.63 to 2.23 \\
\hline Glioma & 796 & $0.005(4)$ & 0.083 & 7.91 & $<0.001$ & 1.0 & 0.06 & 0.02 to 0.17 \\
\hline Abscess & 116 & $0.026(3)$ & 0.083 & 2.22 & 0.026 & 0.8 & 0.31 & 0.08 to 0.96 \\
\hline Tuberculoma & 360 & $0.006(2)$ & 0.083 & 5.30 & $<0.001$ & 1.0 & 0.07 & 0.01 to 0.27 \\
\hline Infarction & 515 & $0.004(2)$ & 0.083 & 6.47 & $<0.001$ & 1.0 & 0.05 & 0.01 to 0.19 \\
\hline
\end{tabular}
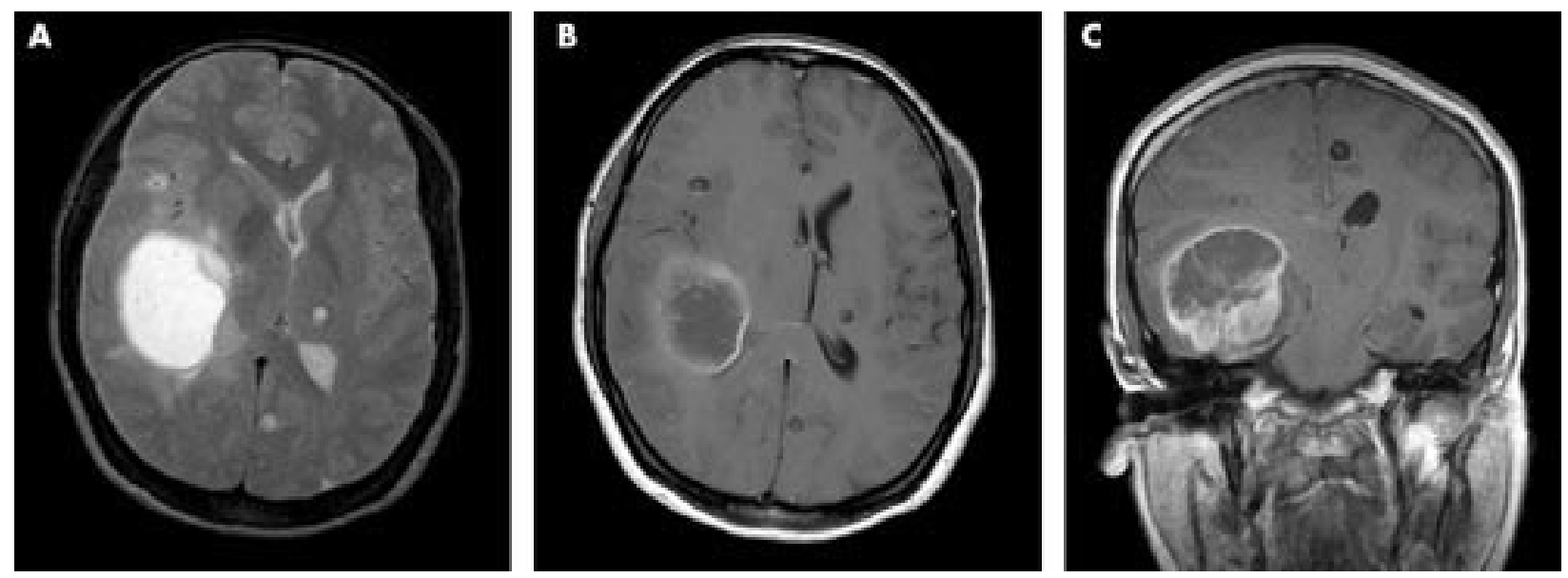

Figure 1 Magnetic resonance imaging in neurocysticercosis with coexistent glioblastoma multiforme. (A) T2 weighted axial section. (B) Postcontrast T1 weighted axial section. (C) Coronal section. The images show multiple cystic lesions with scolex and associated perilesional oedema around a few of the lesions, predominantly in the left hemisphere; the enhancing coexistent lesion is on the right side.

\section{Statistics}

Statistical analysis was done using SPSS software. A z test for proportions and relative risk was used for comparison.

\section{RESULTS}

The overall hospital based disease prevalence of neurocysticercosis was found to be $6.97 \%$, while the prevalence in the control group was $8.3 \%$; the prevalence in cases of dual pathology was just $1.1 \%$. In the Japanese encephalitis subgroup, the prevalence of coexisting neurocysticercosis lesions was the highest at $10.2 \%$, while in other subgroups it was found to be $0.5 \%$ (glioma), $2.6 \%$ (abscess), $0.39 \%$ (infarct), and $0.55 \%$ (tuberculoma). The prevalence of neurocysticercosis was significantly lower overall in patients with dual intracranial pathology than in the control group $(z=11.05, p<0.001)$, though in the Japanese encephalitis subgroup the prevalence was not significantly different from the controls $(z=0.69$, $\mathrm{p}=0.49$ ). In all the other subgroups (glioma, abscess, infarction, and tuberculoma) the prevalence of neurocysticercosis was significantly less than in the controls ( $p<0.05$ for each). For all statistical comparisons, the power of the test was close to 1 in all except for the Japanese encephalitis subgroup, where it was 0.6. The proportion of cases with neurocysticercosis in the overall study population and in the different subgroups, along with the $\mathrm{z}$ and $\mathrm{p}$ values, are as given in table 1 . Relative risk is our best estimate for the relation between neurocysticercosis and other coexistent lesions ( fig 1). The relative risk was largest in Japanese encephalitis, where it was 1.23 (95\% confidence interval 0.63 to 2.23 ). The relative risks and confidence intervals for relations between glioma, abscess, infarct, and tuberculoma are given in table 1. The relative risk for matched pairs was not significant for any of these lesions.
The number of cysts ranged from single to multiple, with single lesions found in 17 of 21 patients, while multiple lesions were found in only four. Most of the cysts were found in the supratentorial compartment except in two patients, where they were infratentorial; all the lesions were intraparenchymal. There was no significant association between the presence of neurocysticercosis in a particular hemisphere and lesion location or severity $(\mathrm{p}=0.413)$. Perilesional oedema, which is a sign of a degenerating or degenerated cyst, was found in nine of 21 patients. Perilesional oedema surrounding cysts did not determine the disease location or severity $(\mathrm{p}=0.623)$.

The subgroup of patients who had neurocysticercosis with Japanese encephalitis was separately evaluated for the above factors. We found that cyst location and surrounding perilesional oedema did not significantly affect the location or severity of the Japanese encephalitis lesions or lead to asymmetry of the lesions $(\mathrm{p}=0.659$ and 0.548 for location and perilesional oedema, respectively). The other subgroups of patients were not evaluated separately as the numbers were too small.

On clinical follow up, nine of the 10 patients with Japanese encephalitis recovered and one died. The patients who had abscess and tuberculoma associated with neurocysticercosis improved after treatment, while three of four patients with glioma died in the follow up period. On histological examination, glioblastoma multiforme was found in all three of these patients, while the fourth had an oligodendroglioma.

\section{DISCUSSION}

The exact prevalence of neurocysticercosis in the Indian population is not known, as most studies in India are hospital 
based. Desai et al found a prevalence rate of $4 \%$ in a necropsy series of patients dying with neuropsychiatric ailments. ${ }^{1}$ However, we found a higher prevalence $(8.3 \%)$ in our control group of hospital patients. This could reflect a difference in the sociodemographic profiles of the patients in the northern India, where our study was done, and in the southern part of India, where Desai's study was done.

The coexistence of neurocysticercosis with various types of intracranial pathology has been reported..$^{1-7}$ Previous reports have also suggested that neurocysticercosis may be a risk factor for various coexisting intracranial lesions such as Japanese encephalitis, glioma, and infarction; and that the immune alterations and a disturbance of the blood-brain barrier caused by neurocysticercosis could be responsible for the coexistence of other lesions. ${ }^{12581011}$

To our knowledge, this is the first controlled MRI based study that sought to determine the association between neurocysticercosis and other common intracranial lesions. In our study, however, we found that the prevalence of neurocysticercosis in cases of dual pathology was only $1.1 \%$ - significantly less than in the control group ( $p<0.0001)$.

The subgroup of patients with Japanese encephalitis had the highest prevalence of neurocysticercosis. Univariate analysis showed that the association in the Japanese encephalitis subgroup was not significantly different from the control population, while in the other subgroups there was evidence of a significantly less common association. Relative risk analysis did not suggest that neurocysticercosis might be a significant risk factor in the pathogenesis of other intracranial lesions, including Japanese encephalitis. The only previously reported MRI study did not have a control population, so a comparison of severity among two groups could not be done, nor could the role of neurocysticercosis as a risk factor be ascertained. ${ }^{5}$ The relatively higher prevalence of neurocysticercosis among patients with Japanese encephalitis may reflect common factors such as pig rearing, poor socioeconomic status, and malnutrition. ${ }^{5}$ Most of the patients in this study came from eastern India, where there is an established endemicity for both Japanese encephalitis and neurocysticercosis. However, the relative risk between the case and control groups was not large enough to establish neurocysticercosis as a risk factor for Japanese encephalitis.

Previous imaging studies using computed tomography and MRI have also suggested a significant local relation between neurocysticercosis with surrounding perilesional oedema and the location and severity of other coexisting lesions. ${ }^{125}$ However, our MRI analysis for the complete study group of coexistent lesions and for Japanese encephalitis alone could not demonstrate such relation in either setting $(\mathrm{p}>0.05)$.

\section{Conclusions}

The coexistence of neurocysticercosis may be an incidental finding in a few patients who are referred from the areas of high prevalence and endemicity and may not imply any association. It seems unlikely that neurocysticercosis is a risk factor for other coexisting lesions. The local presence of neurocysticercosis or surrounding perilesional oedema on MRI in a particular hemisphere does not appear to affect lesion the severity or location of coexisting lesions. However, a prospective study with closely matched controls from defined geographical areas is necessary to validate the results of our study.

\section{Authors' affiliations}

R Azad, R K Gupta, S Kumar, Department of Radiology, Sanjay Gandhi Post-Graduate Institute of Medical Sciences, Lucknow, India C M Pandey, Department of Biostatistics, Sanjay Gandhi Post-Graduate Institute of Medical Sciences

K N Prasad, Department of Microbiology, Sanjay Gandhi Post-Graduate Institute of Medical Sciences

N Husain, Department of Pathology, King George's Medical College, Lucknow, India

M Husain, Department of Neurosurgery, King George's Medical College

Competing interests: none declared

Correspondence to: Dr Rakesh K Gupta, MR Section, Department of Radiodiagnosis, Sanjay Gandhi Post Graduate Institute of Medical Sciences, Lucknow 226014, India; rgupta@sgpgi.ac.in

Received 27 July 2002

Accepted 22 October 2002

\section{REFERENCES}

1 Desai A, Shanker SK, Jayakumar PN, et al. Co-existence of cerebral cysticercosis with Japanese encephalitis: a prognostic modulator. Epidemiol Infect 1997:118: 165-71.

2 Del Brutto $\mathrm{OH}$, Castillo PR, Mena IX, et al. Neurocysticercosis among patients with cerebral gliomas. Arch Neurol 1997; 54:1125-8.

3 Das SK, Nityanand S, Sood K, et al. Japanese B encephalitis with neurocysticercosis. J Assoc Physicians India 1991;39:643-4.

4 Tripathi RP, Gupta A, Gupta S, et al. Co-existence of dual intracranial pathology clinical relevance of proton MRS. Neurol India 2000;48:365-9

5 Singh P, Kalra N, Ratho RK, et al. Coexistent neurocysticercosis and Japanese B encephalitis: MR imaging correlation. Am J Neuroradiol 2001;22:1131-6.

6 Wani MA, Banerii AK, Tandon PN, et al. Neurocysticercosis: some uncommon presentations. Neurol India 1981;29:58-63.

7 Venkatraman S, Nag D, Shukla R. The protean clinical manifestations of neurocysticercosis. In: Proceedings of IVth Annual Conference, UP Chapter of Association of Physicians of India, Varanasi, India, 1982:80-4.

8 Alarcon F, Vanormelingen K, Moncayo J, et al. Cerebral cysticercosis as a risk factor for stroke in young and middle aged people. Stroke 1992:23:1563-5.

9 Del Brutto OH, Rajshekhar V, White AC, et al. Proposed diagnostic criteria for neurocysticercosis. Neurology 2001;57:177-83.

10 Gupta AK, Ayachit VL, Lad VJ, et al. Isohaemagglutinins to human blood group A cells in mice with experimental canine toxocariasis. Indian J Parasitol 1989:13:359-62.

11 Gupta AK, Pavri KM. Alteration in immune response of mice with dual infection of Toxocara canis and Japanese encephalitis virus. Trans $R$ Soc Trop Med Hyg 1987;81:835-40. 